\title{
Spatial pattern formation in chemical and biological systems
}

\author{
Philip K. Maini, ${ }^{a}$ Kevin J. Painter ${ }^{a}$ and Helene Nguyen Phong Chau ${ }^{b}$ \\ ${ }^{a}$ Centre for Mathematical Biology, Mathematical Institute, 24-29 St Giles', Oxford, UK OX1 \\ $3 L B$ \\ ${ }^{b}$ Ecole des Mines de Paris, 60 bd Saint-Michel-75272 Paris Cedex 06, France
}

\begin{abstract}
One of the central issues in developmental biology is the formation of spatial pattern in the embryo. A number of theories have been proposed to account for this phenomenon. The most widely studied is reaction diffusion theory, which proposes that a chemical pre-pattern is first set up due to a system of reacting and diffusing chemicals, and cells respond to this pre-pattern by differentiating accordingly. Such patterns, known as Turing structures, were first identified in chemical systems only recently. This article reviews the application of reaction diffusion theory to chemical systems and then considers a number of biological applications.
\end{abstract}

One of the central issues in developmental biology is the understanding of the emergence of structure and form from the almost uniform mass of dividing cells that constitutes the early embryo. Although genes play a key role, genetics says nothing about the actual mechanisms which bring together the constituent parts into a coherent pattern-knowing the dictionary does not mean that we know Shakespeare.

In his seminal paper, Turing ${ }^{1}$ demonstrated, theoretically, that a system of reacting and diffusing chemicals could spontaneously evolve to spatially heterogeneous patterns from an initially uniform state in response to infinitesimal perturbations. Remarkably, he showed that diffusion could drive a chemical system to instability, leading to spatial pattern where no prior pattern existed. He specifically considered a system of two chemicals, in which one was an activator and the other an inhibitor. That is, one chemical, the activator, stimulated and enhanced the production of the other chemical, which, in turn, depleted or inhibited the formation of the activator. $\mathrm{He}$ showed that if the diffusion of the inhibitor was greater than that of the activator, then diffusion-driven instability could result. This is counter-intuitive, as one usually thinks of diffusion as a homogenizing process.

To gain an intuitive understanding of diffusion-driven instability, let us consider the following analogy: suppose that we have the simple autocatalytic process $\mathrm{A}+\mathrm{B} \rightarrow 2 \mathrm{~B}$ in an unstirred reactor initially full of chemical $A$ but with no chemical B. Then, obviously, there will be no reaction. If we now seed the reaction domain with some $B$ at various local sites and if A can diffuse but B is immobilised, reaction will only occur where there has been seeding, with high concentrations of B building up at these points. Eventually, A would disappear and we would be left with 'spots' of B. If, however, there is a supply of A across the domain (perhaps by formation from a precursor) and also a decay step for B to limit its growth, then it may be possible to get a balance between supply and diffusion away of A balancing the decay of B in the spots, to give steady-state, long-lived pattern, with high A concentrations in between the spots and high B concentration in the spots. The exact pattern of spots would still depend on the precise initial seeding, but Turing makes two remarkable predictions: first, that this structure develops spontaneously even from an initially almost homogeneous distribution of $\mathrm{A}$ and $\mathrm{B}$ provided that $\mathrm{A}$ diffuses more rapidly than $\mathrm{B}$ and, second, that the final pattern does not necessarily depend on the initial perturbation if $\mathrm{B}$ has a non-zero diffusivity-the spots adjust their position to the demands of the local 'supply and demand' due to diffusion and reaction.
A general reaction diffusion (RD) system has the form

$$
\frac{\partial \boldsymbol{u}}{\partial t}=D \nabla^{2} \boldsymbol{u}+\boldsymbol{f}(\boldsymbol{u}, \boldsymbol{p})
$$

where $\boldsymbol{u}$ is a vector representing chemical concentrations. Boundary conditions are usually taken as zero flux, that is, the domain boundary is assumed impermeable to the chemicals. Typically, one considers a two-chemical system, in which case $\boldsymbol{u}=\left(u_{1}, u_{2}\right)$, where $u_{1}$ and $u_{2}$ are chemical concentrations. The first term on the right-hand side represents diffusion, with $D$ a matrix of diffusion coefficients, assumed constant, and the second term represents chemical reactions, with kinetic parameters $\boldsymbol{p}$, for example, rate constants, production and degradation terms. The form of $\boldsymbol{f}$ depends on the system being studied (see later) but here we give an illustrative example due to Schnakenberg: ${ }^{2}$

Consider the series of reactions

$$
\mathrm{X} \rightleftharpoons \mathrm{A}, \quad 2 \mathrm{X}+\mathrm{Y} \rightarrow 3 \mathrm{X}, \quad \mathrm{B} \rightarrow \mathrm{Y}
$$

Using the law of mass action, we have that the production of $\mathrm{X}$ occurs at the rate

$$
k_{2} a-k_{1} u_{1}+k_{3} u_{1}{ }^{2} u_{2}
$$

while that of $\mathrm{Y}$ is

$$
k_{4} b-k_{3} u_{1}{ }^{2} u_{2}
$$

where $u_{1}, u_{2}, a$ and $b$ are the concentrations of $\mathrm{X}, \mathrm{Y}, \mathrm{A}$ and $\mathrm{B}$, respectively, and $k_{1}, \ldots, k_{4}$ are rate constants. Assuming that $\mathrm{X}$ and $\mathrm{Y}$ diffuse with diffusion coefficients $D_{1}$ and $D_{2}$, respectively, and that $\mathrm{A}$ and $\mathrm{B}$ are in abundance so that $a$ and $b$ can be assumed approximately constant, the reaction diffusion system satisfied by $u_{1}$ and $u_{2}$ is

$$
\begin{aligned}
& \frac{\partial u_{1}}{\partial t}=D_{1} \nabla^{2} u_{1}+k_{2} a-k_{1} u_{1}+k_{3} u_{1}^{2} u_{2} \\
& \frac{\partial u_{2}}{\partial t}=D_{2} \nabla^{2} u_{2}+k_{4} b-k_{3} u_{1}^{2} u_{2}
\end{aligned}
$$

A uniform steady state of a general RD system is the vector of concentrations $\boldsymbol{u}_{0}$, constant in space and time, satisfying $\boldsymbol{f}\left(\boldsymbol{u}_{0}, \boldsymbol{p}\right)=0$. Turing showed that, under certain conditions on the kinetic parameters and diffusion coefficients, such a steady state, stable in the absence of diffusion, could be driven unstable in the presence of diffusion and evolve to a spatially patterned state as a certain parameter - the bifurcation, or control, parameter-was varied. Turing was interested in morphogenesis, the formation of structure, so he proposed that 
this chemical pattern could serve as a pre-pattern to which cells would respond in such a way that a spatial structure would form. For example, if one of the chemicals was a plant growth hormone, then the pre-pattern could result in patterned growth. More generally, these chemicals (termed morphogens) could trigger a genetic switch causing cell differentiation.

Since Turing's paper there has been a vast literature on both the theoretical and practical aspects of RD systems (see, for example, ref. 3-8). Turing models have been applied to such diverse areas as ecology, ${ }^{9}$ semiconductor physics, ${ }^{10}$ material sciences ${ }^{11,12}$ hydrodynamics, ${ }^{13}$ astrophysics ${ }^{14}$ and even economics. ${ }^{15}$

In this partial review we focus only on two specific areas of application, namely chemistry and biology. The application of Turing's theory to these areas has come in for much criticism, for three main reasons: first, there was no conclusive proof that Turing patterns actually existed in nature; second, there is no definitive proof of the existence of morphogens; third, the solutions tend to be very sensitive to perturbations and thus the model appears unrealistic when applied to many examples in morphogenesis where the patterning process is robust. Although the evidence for the existence of morphogens is still tenuous, Turing structures have now been found in chemistry, and different types of boundary conditions have been shown to increase the stability of model solutions. We begin by a brief survey of recent work on the identification of Turing patterns in chemistry.

\section{Spatial and spatiotemporal patterns in chemistry}

Time-dependent patterns in chemical systems have been studied for many years. The best known oscillatory reaction is the Belousov-Zhabotinsky reaction, first reported by Belousov $^{16}$ in an unpublished paper which appeared in the book edited by Field and Burger ${ }^{17}$ (see Plate 1). This is an example of a chemical oscillator. Briefly, bromate ions oxidise malonic acid in a reaction catalysed by cerium, which has the states $\mathrm{Ce}^{3+}$ and $\mathrm{Ce}^{4+}$. Sustained periodic oscillations are observed in the cerium ions. If, instead, one uses the catalyst $\mathrm{Fe}^{2+}$ and $\mathrm{Fe}^{3+}$ and phenanthroline, the periodic oscillations are visualized as colour changes between reddish-orange and blue ${ }^{19}$ (see Plate 1). In this system, the activator is $\mathrm{HBrO}_{2}$ and the inhibitor is $\mathrm{Br}^{-}$. The model and detailed analysis can be found in ref. 20 (see also ref. 21-23). This system also exhibits a number of different types of wave structures, such as propagating fronts, spiral waves, target patterns and toroidal scrolls. ${ }^{24-30}$

Until recently, however, attempts to obtain experimental evidence of Turing structures had proved unsuccessful. A key reason for the failure to find Turing patterns in experiments was due to the need for significant variation in diffusion coefficients of the reactants. Turing structures require the inhibitor to diffuse at a much faster rate than the activator. Numerical simulations of many of the model systems studied, such as the Brusselator model or the reduced Oregonator model of the Belousov-Zhabotinsky reaction, ${ }^{31,32}$ predict that Turing structures are only generated when diffusion coefficients differ by at least one order of magnitude, that is, $D_{\mathrm{HBrO}_{2}} \ll D_{\mathrm{Br}^{-}}$. Experimentally obtained diffusion coefficients of the reactants, however, show much less variation. More recently, it has been shown $^{33}$ that a diffusion induced instability can occur under certain very special conditions with nearly equal diffusion coefficients.

Note that thermodynamically closed systems must eventually approach their unique state of chemical equilibrium, which will be spatially homogeneous. In such systems, any pattern formation is necessarily transient.

The first experimental evidence of the existence of Turing structures was presented by De Kepper's group in Bord- (a)

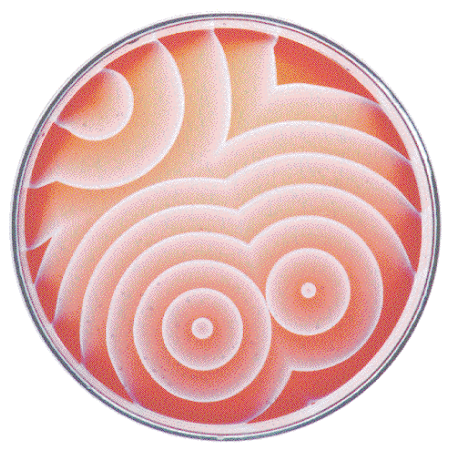

(b)

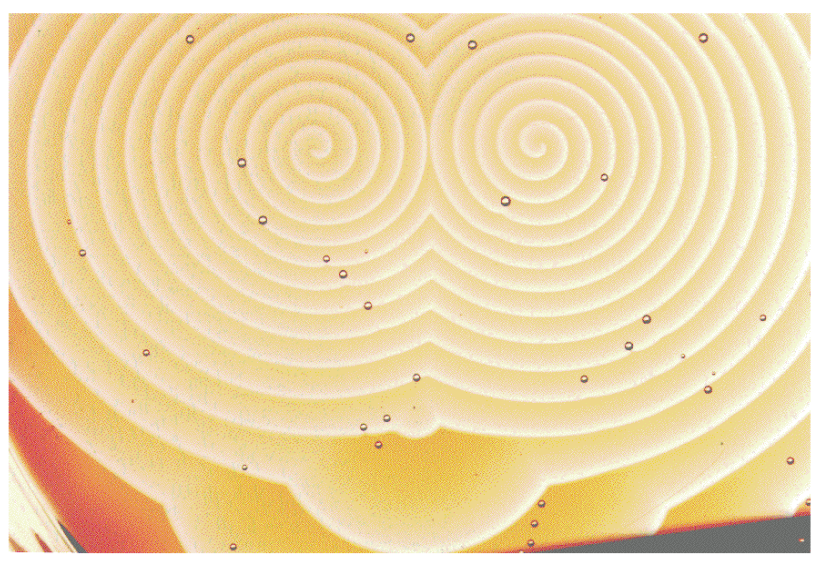

Plate 1 Spatiotemporal patterns in the Belousov-Zhabotinsky reaction. (a) Target patterns, $(b)$ spiral patterns (Reprinted from ref. 18 with kind permission from The Royal Society of Chemistry).

eaux. ${ }^{34,35}$ They used an open reactor system, wherein reactants were continuously fed into the system, keeping it far from equilibrium. The resultant sustained standing nonequilibrium chemical patterns exhibit all the properties of Turing structures. The verification of Turing's prediction, nearly forty years after the original theory, has revived interest and motivated greater study in the area.

The reaction that has received the greatest attention is the chlorite-iodide-malonic acid-starch (CIMA) reaction ${ }^{36}$ and we now examine this is some detail.

\section{The chlorite-iodide-malonic acid-starch reaction (CIMA reaction)}

The reactor consists of a block of hydrogel in contact with the contents of two continuous-flow stirred tank reactors (CSTRs) on opposite sides of the gel. Each of the reservoirs contains a non-reacting subset of reagents which are continuously pumped into the reaction. This keeps reactant concentrations uniform and constant during the experiment. The gel inhibits any convective motion so that the only motion involved is due to diffusion of the chemicals. Full details of the experimental setup to generate stationary patterns can be found in ref. 35-37.

A soluble starch colour indicator is included to visualise concentration changes. Starch colour switches from yellow to blue, which is monitored in transmitted light with a video camera. Dark blue regions correspond to high concentrations of the starch-triiodide complex whereas clear zones are characterized by the presence of oxidised states only. Note that starch was added simply to aid visualisation, but its binding to the iodide ions turned out to be absolutely crucial, as it 
reduced the mobility of the latter and hence enhanced the ratio of diffusion coefficients allowing Turing instability to occur (see later).

Three geometries of reactors can be distinguished.

(1) The thin strip reactor. Used in the original experiments, this is a narrow rectangular ribbon of a transparent chemically inert hydrogel with a thickness greater than five times the wavelength of the pattern. Reactors have been devised with a thickness of the order of the pattern wavelength to approximate a two-dimensional system.

(2) Ouyang and Swinney ${ }^{38}$ use a flat disk of gel sandwiched between two porous glass disks.

(3) To study the influence of geometry on the pattern selection mechanism, De Kepper et $\mathrm{al}^{35}$ have designed a reactor with an annular gel strip. Two different experiments were performed, one in the classical rectangular strip and the other in the annular reactor. It was found that generation of the patterns was not influenced by the geometry of the reactor.

De Kepper et $\mathrm{al}^{35}$ have observed the formation of a stationary three-dimensional pattern consisting of parallel lines of periodic spots. This body-centred cubic structure is in agreement with the theory developed in ref. 39. Using symmetry arguments, this theory demonstrated that the only Turing structures that persist in three-dimensional space are those with wave-vectors satisfying a definite angular relation. The body-centred cubic pattern is found to be the most stable. Patterns develop spontaneously over a well defined range of malonic acid concentration. The wavelength of $0.2 \mathrm{~mm}$ appears intrinsic and is not related to geometric characteristics of the system. The pattern is also influenced by the temperature, which controls the reaction rates.

Ouyang and Swinney ${ }^{37}$ obtained quasi two-dimensional structures, maintained indefinitely in a non-equilibrium state. The patterns develop spontaneously from the spatially uniform state on variation of a control parameter (chemical concentrations or temperature). These Turing patterns are shown in Fig. 1. In these experiments, the concentrations of iodide and malonic acid are chosen as control parameters and other concentrations are kept constant. Initially, transient yellow circles emerge and start to grow in a blue surrounding. These structures dissolve and break into dot patterns demonstrating a wide distribution of sizes. The dots evolve to a
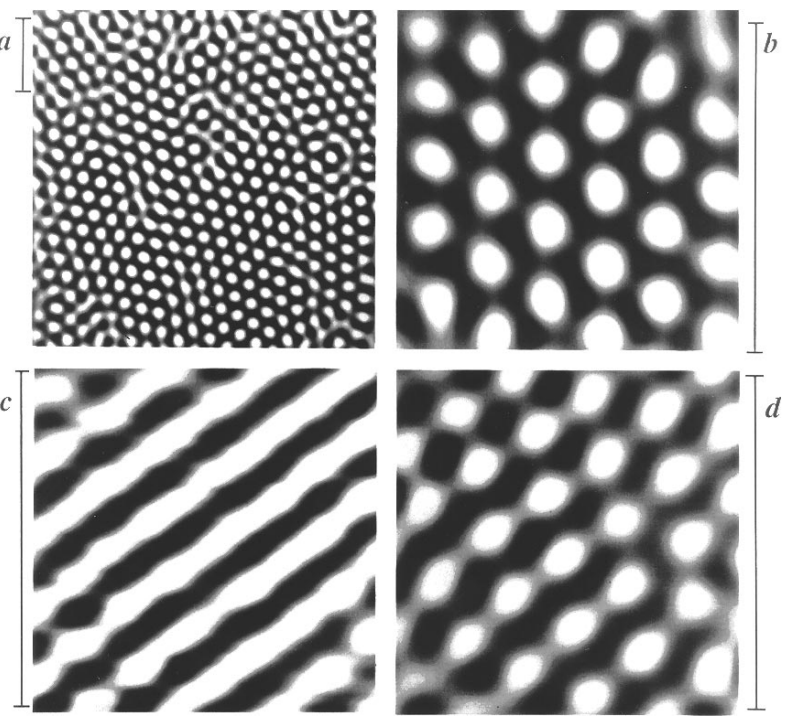

Fig. 1 Different types of stationary chemical (Turing) patterns in the CIMA reaction (see ref. 37 for full details). Bars represent $1 \mathrm{~mm}$. (Reprinted with permission from Nature.) quasi-stationary structure consisting of multiple domains of yellow hexagons of different orientations. These hexagons, denoted $H_{\pi}$, are approximately uniform in size.

Re-entrant hexagons, that is dark-blue hexagons formed in a light background, designated by $H_{0}$, and distributed in a structure similar to a honeycomb lattice, have recently been reported in ref. 40, but these patterns appear only transiently.

At high iodide or low malonic acid concentration the domains are no longer composed of hexagons, but a stationary array of regularly spaced out stripes of equal width forms. A stable mixed solution of the above two patterns can also occur. These patterns can be qualitatively described as the stretching of the regular hexagonal lattice along the direction of the stripes. Distorted yellow dots are no longer uniform in size or form. A transition from an initially uniform state to a hexagonal pattern is also obtained through a temperature change.

A rhombic pattern has been generated in ref. 41 using special initial conditions. The photosensitive nature of the CIMA reaction is exploited by imposing particular initial conditions using illuminated, computer-generated photographs of specific spatial patterns. The desired perturbation is thus created in the reaction medium and once this initial pattern has settled down, the system is left to evolve freely. The ultimate pattern that arises depends on the characteristic angle of the imposed rhombic initial perturbation.

A more complex structure, referred to as a 'black-eye' pattern and illustrated in Fig. 2, appears well beyond the primary instability of a hexagonal array through increase in the malonic acid concentration. ${ }^{41}$

This black-eye pattern is thought to be the result of a resonant interaction between the basic modes of a hexagonal array. It can be seen as the superposition of two different hexagonal lattices: one of large yellow spots and another composed of small dark blue dots. At a higher concentration of malonic acid, the black-eye pattern becomes unstable and a transition to regularly spaced stripes of equal width occurs.

The spatial patterns produced by this reaction are fundamentally different to the more widely known target and spiral 'patterns'. The latter are not really patterns but are actually wave structures. We now briefly consider two other types of patterns.

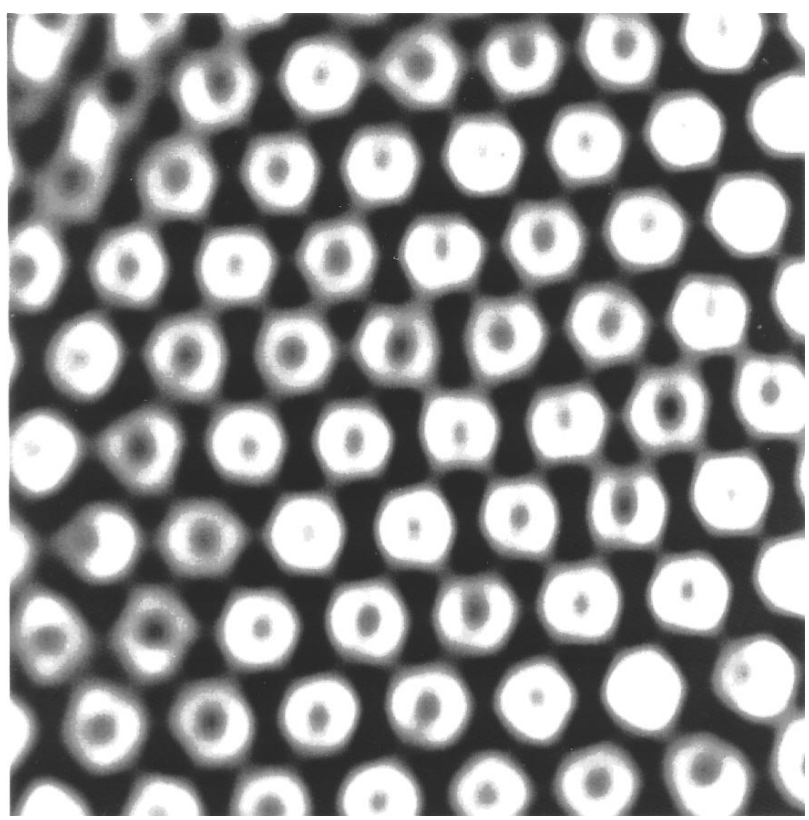

Fig. 2 An example of the 'black-eye' patterns generated in ref. 41. (Reprinted with kind permission from ref. 41.) 
The polyacrylamide-Methylene Blue-sulfide-oxygen system

Turing-like patterns have been observed in the polyacrylamide-Methylene Blue-sulfide-oxygen (PA-MBO) reaction. ${ }^{42}$ As this is a closed setup only transient patterns are possible and the system finally relaxes to its equilibrium state. Details of this reaction and corresponding mathematical models can be found in ref. 43-45.

Briefly, this reaction exploits the oscillating properties of the Methylene Blue-sulfide-oxygen (MBO) system, which has been studied by Burger and Field. ${ }^{43}$ The reaction of this oscillator is the Methylene Blue catalysed oxidation of $\mathrm{HS}^{-}$by $\mathrm{O}_{2}$ in water at $\mathrm{pH}=12$. The monomer Methylene Blue exhibits two stable forms: the blue $\mathrm{MB}^{+}$and the colourless reduced form $\mathrm{MBH}$. The redox reaction linking these two forms can be represented in two steps:

$$
\begin{aligned}
\mathrm{MB}^{+}+\mathrm{e}^{-} & \rightleftharpoons \mathrm{MB}^{0} \\
\mathrm{MB}^{0}+\mathrm{e}^{-}+\mathrm{H}^{+} & \rightleftharpoons \mathrm{MBH}
\end{aligned}
$$

Resch et al $^{44}$ determined a mechanism for the $\mathrm{MBO}$, composed of fourteen reactions involving several radical and anionic compounds. A reduced model consisting of eight chemical reactions and five variables has been proposed by Zhang and Field. ${ }^{45}$

The experiment is performed in a Petri dish and is closed with respect to all chemical species except oxygen. This effect means that only transient patterns are possible - the system relaxes to its equilibrium state. Transient patterns show a hexagonal colourless lattice in a dark-blue surround. Removal of the gel from the Petri dish allows the surfaces contact with the Methylene Blue solution and oxygen. This allows oxygen to diffuse from below into the gel. Short-lived blue hexagons appear in a light-blue background. The pattern relatively quickly disintegrates to the homogeneous state. Colourless spots eventually re-emerge from this uniform state after another $50 \mathrm{~min}$. If the gel is not removed from the Petri dish, the colourless hexagons break up to form short stripes and zigzag patterns. This spontaneous transition from hexagonal to zigzags originates through modification of the reactants concentrations which inevitably occurs in a closed system. The zigzag pattern is found to coexist with the hexagonal structure. Experimental results are shown in Fig. $3 .^{42}$

The hexacyanoferrate(II)-iodate-sulfite reaction

Lee et $a l .{ }^{46}$ have observed non-Turing stationary patterns in the hexacyanoferrate(II)-iodate-sulfite (FIS) reaction, using an experimental technique similar to that used for the CIMA reaction. These patterns develop through propagation of chemical fronts from the initial perturbation. The fronts propagate towards one another but stop when they reach a criti-
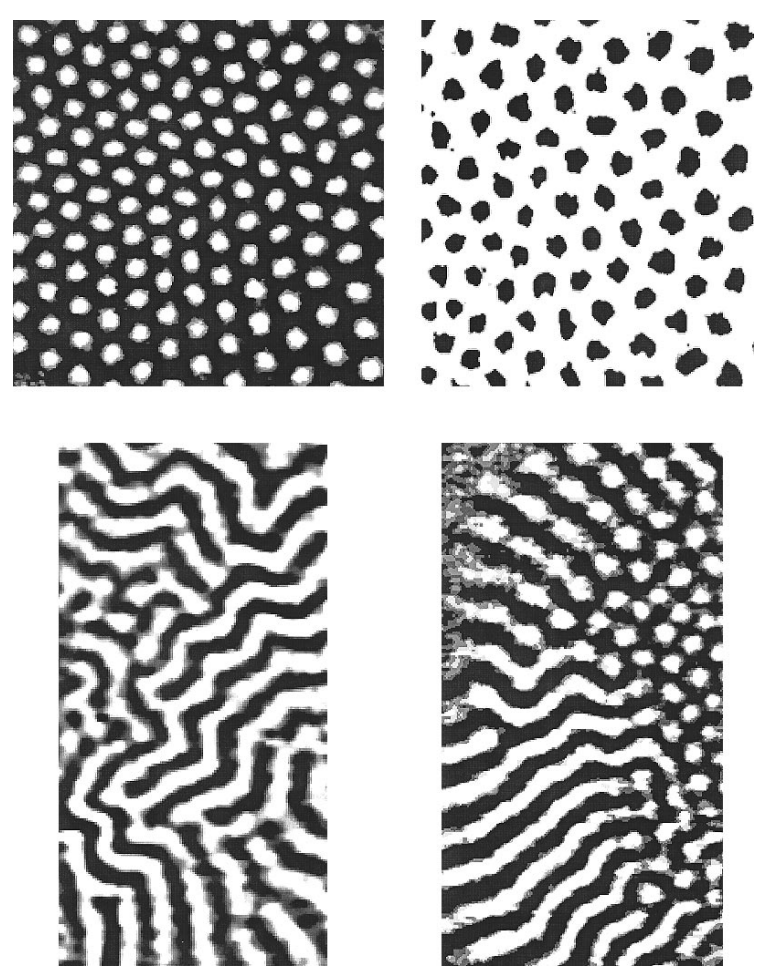

Fig. 3 Transient Turing-like patterns in the PA-MBO system (see ref. 42 for full details). (Reprinted with kind permission from ref. 42.)

cal distance. A typical pattern is shown in Fig. 4. The patterns have no long-range regularity, making them fundamentally different to patterns developed through a Turing bifurcation.

The reaction also exhibits 'self-replicating' spots. ${ }^{47}$ In a pattern of this form a spot will grow, divide into two and the two spots move away. As they fill the domain, some spots decay. In addition to spots, growing annuli have also been observed. ${ }^{47} \mathrm{~A}$ brief mathematical analysis of this phenomenon has been carried out in ref. 48 for the Gray-Scott model. ${ }^{49-51}$

More recently, Haim et al. ${ }^{52}$ have observed oscillating spots in the FIS reaction. This behaviour is thought to arise through the interactions of the chemical front with the reactor boundary. Mathematical analysis of a bistable RD model exhibiting patterning phenomena similar to that observed in the FIS reaction supports this suggestion.

Lengyel and Epstein ${ }^{53}$ have given an account of some of the difficulties in creating Turing patterns in experimental systems and how new systems can be designed to create such patterns. Recently they have examined the challenge of obtaining
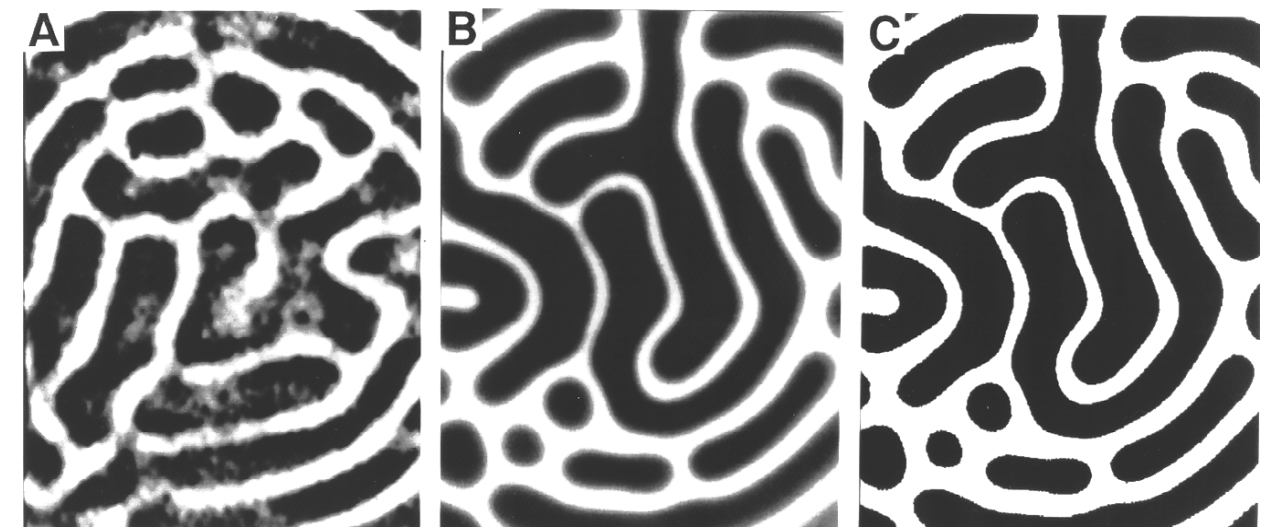

Fig. 4 Time asymptotic patterns for the FIS reaction. Dark regions correspond to low hexacyanoferrate(II) concentration and light regions to high hexacyanoferrate(II) concentration. ${ }^{46}$ (Reprinted with kind permission from ref. 46.) 
Turing patterns in a closed system at room temperature. ${ }^{54}$ Such a system would be of significance in lecture demonstrations of Turing systems and complimentary to the BelousovZhabotiinsky reaction, which is comparatively easy to demonstrate.

\section{Modelling spatial and spatiotemporal phenomena}

Turing considered linear reaction kinetics. Although this is unrealistic chemically, and predicts unbounded growth in chemical concentrations, it enabled him to carry out a mathematical analysis and predict the types of patterning phenomena that the model could exhibit. There are two types of patterns: spatial patterns, wherein a spatially heterogeneous pattern emerges with a defined wavelength; and spatiotemporal patterns, in which the pattern varies periodically in time, as well as having a defined spatial wavelength. Such patterns emerge when the uniform steady state loses stability as a control bifurcation parameter passes through a critical value. At that critical value, the wavelength of the pattern and its period (in the case of spatiotemporal patterns) can be calculated in terms of the parameters of the model. Which type of pattern emerges depends on the parameter space being considered.

Since Turing's paper, several RD models have been considered with non-linear kinetic terms. These models are more realistic in that their solutions evolve to bounded values, and are derived essentially in three different ways: (i) phenomenologically, (ii) to model a hypothetical reaction and (iii) empirically. An example of (i) is the model of Geirer and Meinhardt. ${ }^{55}$ One version of this model has the kinetics $f_{1}\left(u_{1}\right.$, $\left.u_{2}\right)=k_{1}-k_{2} u_{1}+\left(k_{3} u_{1}{ }^{2} / u_{2}\right), \quad f_{2}\left(u_{1}, u_{2}\right)=k_{4} u_{1}{ }^{2}-k_{5} u_{2}$, where $k_{1}, \ldots, k_{5}$ are positive constants. In this system, $u_{1}$ is the activator; it is produced by autocatalysis and it activates the production of $u_{2}$, which is the inhibitor, inhibiting the production of $u_{1}$.

The Schnakenberg ${ }^{2}$ model discussed above is an example of (ii) and is based on a cubic autocatalytic process.

An example of (iii) is the Thomas model, ${ }^{56}$ in which $f_{1}\left(u_{1}\right.$, $\left.u_{2}\right)=k_{1}-k_{2} u_{1}-H, f_{2}\left(u_{1}, u_{2}\right)=k_{3}-k_{4} u_{2}-H$, where $H=$ $k_{5} u_{1} u_{2} /\left(k_{6}+k_{7} u_{1}+k_{8} u_{1}{ }^{2}\right) u_{2}$, and $k_{1}, \ldots, k_{8}$ are positive parameters. This is based on a specific reaction involving the substrates oxygen $\left(u_{1}\right)$ and uric acid $\left(u_{2}\right)$, which react in the presence of the enzyme uricase.

The patterning properties of these models have been well documented for one-dimensional domains with zero flux boundary conditions in the vicinity of primary bifurcation points. However, the behaviour of solutions far away from bifurcation points, or under different types of boundary conditions, has received little attention. Until recently, the twodimensional pattern forming properties of these systems had also received little attention. However, the experimental observations reviewed in the previous section have resulted in a great deal of analysis of these systems in two dimensions. In some cases, standard RD systems have been examined to see if they exhibit solutions similar to those observed experimentally. For example, Dufiet and Boissonade ${ }^{57}$ have derived a variety of two-dimensional patterns using Schnakenberg chemical kinetics that are similar to those observed in the CIMA reaction (Fig. 5). Their numerical simulations also predict rhombic arrays. A theoretical study of pattern formation in the presence of symmetries has been presented by Gunaratne et $a l^{41}$ who have demonstrated the existence of rhombic arrays and derived their stability properties. The theoretical prediction is in good qualitative agreement with experimental prediction of stability.

In other cases, specific models have been developed for particular reactions and then solved numerically. For example, the following empirical rate law model for the CIMA reaction has been proposed by Lengyel and co-workers. ${ }^{58-60}$
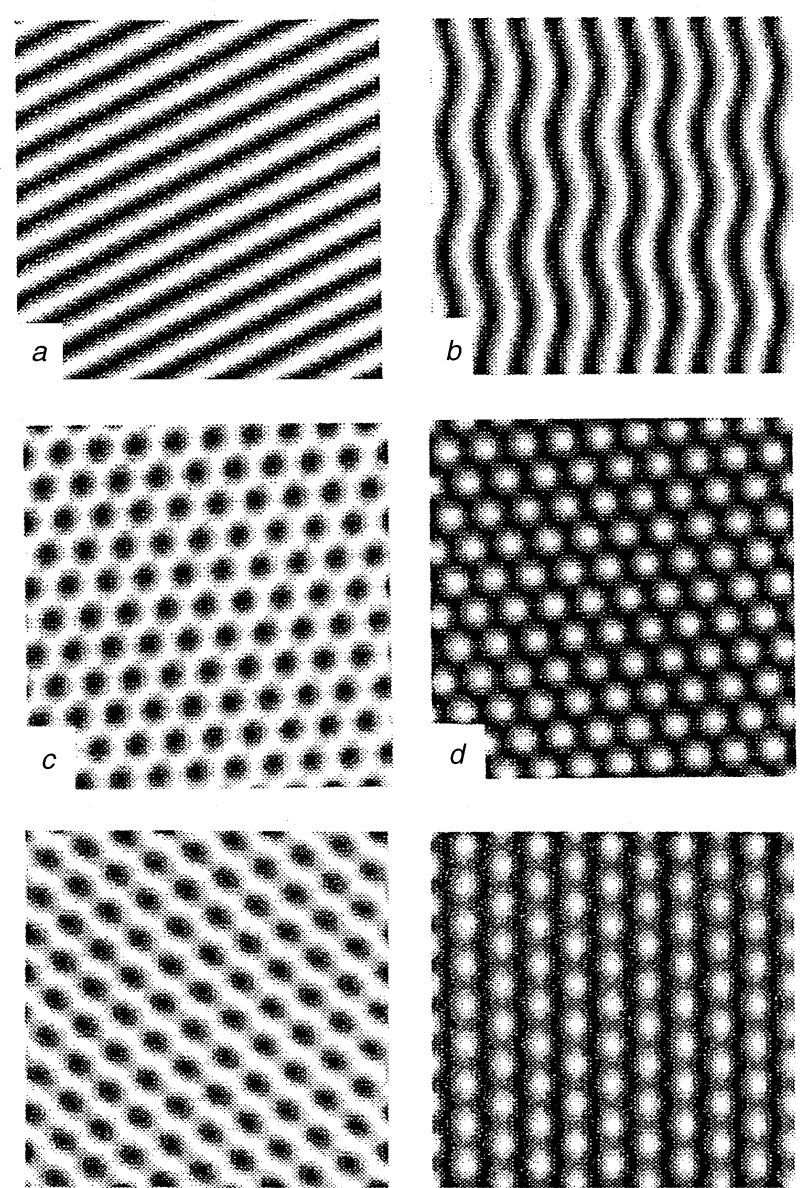

Fig. 5 Patterns generated by the Schnakenberg model. ${ }^{57}$ (Reprinted with kind permission from ref. 57.)

\section{Modelling of the CIMA reaction}

The model proposed by Lengyel and Epstein stresses three processes: the reaction between malonic acid (MA) and iodine to create iodide, the reaction between chlorine dioxide and iodide, and the reaction between chlorite and iodide. The model has the form:

$$
\begin{gathered}
\mathrm{MA}+\mathrm{I}_{2} \rightarrow \mathrm{IMA}+\mathrm{I}^{-}+\mathrm{H}^{+} \quad r_{1}=\frac{k_{1}[\mathrm{MA}]\left[\mathrm{I}_{2}\right]}{w_{1}+\left[\mathrm{I}_{2}\right]} \\
\mathrm{ClO}_{2}+\mathrm{I}^{-} \rightarrow \mathrm{ClO}_{2}{ }^{-}+\frac{1}{2} \mathrm{I}_{2} \quad r_{2}=k_{2}\left[\mathrm{ClO}_{2}\right]\left[\mathrm{I}^{-}\right] \\
\mathrm{ClO}_{2}{ }^{-}+4 \mathrm{I}^{-}+4 \mathrm{H}^{+} \rightarrow \mathrm{Cl}^{-}+2 \mathrm{I}_{2}+2 \mathrm{H}_{2} \mathrm{O} \\
r_{3}=k_{3 \mathrm{a}}\left[\mathrm{ClO}_{2}^{-}\right]\left[\mathrm{I}^{-}\right]\left[\mathrm{H}^{+}\right]+k_{3 \mathrm{~b}} \frac{\left[\mathrm{ClO}_{2}^{-}\right]\left[\mathrm{I}_{2}\right]\left[\mathrm{I}^{-}\right]}{w_{3}+\left[\mathrm{I}^{-}\right]^{2}}
\end{gathered}
$$

where [] denote chemical concentration and $k_{1}, k_{2}, k_{3 \mathrm{a}}, k_{3 \mathrm{~b}}$, $w_{1}$ and $w_{3}$ are positive constants. Parameter values can be determined experimentally. ${ }^{58,59}$

The reaction rates $r_{1}, r_{2}$ and $r_{3}$ are derived empirically and lead to a set of five coupled differential equations, with five variables corresponding to the concentration $[\mathrm{MA}],\left[\mathrm{I}_{2}\right]$, $\left[\mathrm{ClO}_{2}\right],\left[\mathrm{I}^{-}\right]$and $\left[\mathrm{ClO}_{2}^{-}\right]$.

Numerical simulation of the above system yields oscillatory behaviour in close agreement with that observed experimentally. ${ }^{53,61}$

Numerical and theoretical analysis of the full, five-variable model with spatial (diffusive) terms is a computationally demanding task. As a first approximation, a more manageable two variable model has been derived ${ }^{60}$ by making the experimentally realistic assumption that the concentrations of malonic acid, chlorine dioxide and iodine are constant.

A key problem faced when obtaining experimental verification of Turing structures is the required variation of diffusion 
coefficients. Diffusion of the inhibitor must be larger than diffusion of the activator for Turing instabilities to develop. The reactants above, however, have similar diffusion rates.

Lengyel and Epstein $^{60}$ have suggested that reactions occurring between the iodide and the gel or starch may serve to reduce the diffusion rate of the activator. They demonstrate mathematically (see below) how an effect of this nature may serve to increase the ratio of diffusion coefficients sufficiently for Turing structures to develop and apply this approach to the CIMA reaction. The model, referred to as the LengyelEpstein or 'Brandeisator' model is

$$
\begin{aligned}
& \frac{\partial u_{1}}{\partial t}=k_{1}-u-\frac{4 u_{1} u_{2}}{1+u_{1}^{2}}+\nabla^{2} u_{1} \\
& \frac{\partial u_{2}}{\partial t}=k_{2}\left[\mathrm{k}_{3}\left(u_{1}-\frac{u_{1} u_{2}}{1+u_{1}^{2}}\right)+c \nabla^{2} u_{2}\right]
\end{aligned}
$$

where $u_{1}, u_{2}$ are the concentrations of iodide and chlorite, respectively, and $k_{1}, k_{2}, k_{3}$ and $c$ are positive constants. Numerical simulations of the above model under realistic parameter values yield diffusion-driven stationary patterns with a wavelength calculated at $c a .0 .15 \mathrm{~mm}$. This is close to the experimental wavelength of $0.2 \mathrm{~mm}$. Numerical simulations of the above model demonstrate Turing structures qualitatively similar to the structures observed in the experimental system. $^{62}$

The effect of starch and/or the gel has been confirmed in several experiments. For example, in experiments where no reaction occurs between gel and iodine the presence of starch is essential for the development of structures. Details of the various reactions can be found in ref. 63-65.

At a low starch concentration the reaction demonstrates travelling wave trains as opposed to Turing structures. This suggests that the system has been shifted to the oscillatory state. Indeed, this is as we should expect, as in the absence of spatial diffusion the model predicts oscillatory behaviour. The effect of the starch is to shift the reaction from oscillatory behaviour to Turing structures. This idea has enabled investigators to examine the transition from oscillatory to Turing structures. $^{66}$

\section{Modification of the diffusion ratio}

As mentioned above, a key problem in the verification of Turing structures is the required variation of diffusion coefficients. For a general reaction diffusion system, the ratio may be changed as follows: ${ }^{67}$ consider a standard two-species reaction diffusion system of the form

$$
\begin{aligned}
& \frac{\partial u}{\partial t}=f(u, v)+D_{u} \nabla^{2} u, \\
& \frac{\partial v}{\partial t}=g(u, v)+D_{v} \nabla^{2} v .
\end{aligned}
$$

We make the additional assumption that the activator is involved in a reaction of the form:

$$
\mathrm{U}+\mathrm{S} \rightleftharpoons \mathrm{C}
$$

Assuming that both $\mathrm{S}$ and $\mathrm{C}$ are immobile, the RD system is now modified to:

$$
\begin{aligned}
& \frac{\partial u}{\partial t}=f(u, v)-r_{1} u s+r_{2} c+D_{u} \nabla^{2} u \\
& \frac{\partial v}{\partial t}=g(u, v)+D_{v} \nabla^{2} v \\
& \frac{\partial c}{\partial t}=r_{1} u s-r_{2} c
\end{aligned}
$$

where $s$ and $c$ are the concentrations of $\mathrm{S}$ and $\mathrm{C}$, respectively, and $r_{1}\left(r_{2}\right)$ is the forward (reverse) rate of reaction in the new reaction. If $r_{1}$ and $r_{2}$ are large, then using singular perturbation theory, $c$ can be approximated in terms of $u$ by $c \equiv r u$, where $r=s_{0} r_{1} / r_{2}$ and we have assumed that the concentration of $\mathrm{S}$ remains close to its initial value, $s_{0}$.

On addition of the first and third equations above, we obtain the following equation for the activator:

$$
(1+r) \frac{\partial u}{\partial t}=f(u, v)+D_{u} \nabla^{2} u
$$

Thus when $r \gg 1$ the diffusion of the activator is greatly reduced.

This demonstrates how the formation of an immobile complex can reduce the effective diffusion rate of the activator species. It was this type of approach that was first used by Lengyel and Epstein ${ }^{53,60}$ to explain how Turing structures develop in the CIMA reaction. In this case, starch forms a stable complex with triiodide ions via the reaction

$$
\mathrm{S}+\mathrm{I}^{-}+\mathrm{I}_{2} \rightleftharpoons \mathrm{SI}_{3}{ }^{-} \quad K=\frac{\left[\mathrm{SI}_{3}{ }^{-}\right]}{[\mathrm{S}]\left[\mathrm{I}_{2}\right]\left[\mathrm{I}^{-}\right]}
$$

and the high molecular weight of the complex reduces the rate of diffusion.

\section{Verification of the wavelength rule in Turing systems}

A characteristic of Turing patterns is the intrinsic relationship between the average diffusion coefficient of the reactants and the wavelength of the pattern. This characteristic differentiates Turing patterns from other patterning phenomena. Turing demonstrated that near the bifurcation from a uniform steady state to Turing patterns, the wavelength of the pattern is predicted to be $(2 \pi T D)^{1 / 2}$, where $D=\left(D_{1} D_{2}\right)^{1 / 2}$, and $D_{1}, D_{2}$ are the diffusion coefficients. $T$ is the period of the limit cycle when the system is at the onset of Hopf bifurcations (temporally varying pattern).

Using two types of gel, and varying the concentrations of the gels, it is possible to test experimentally if Turing's rule is obeyed for the CIMA reaction. ${ }^{68}$ With the above variations, pattern wavelengths can be measured when $D$ is varied over a factor of three. The corresponding plot of average diffusion coefficient against the experimental wavelength confirms Turing's prediction. Experimental measurement of the period of limit cycles at the onset of Hopf bifurcations is also in good agreement with the theoretical predictions.

\section{Other models}

Pearson ${ }^{69}$ has performed numerical simulations on the model of Gray and Scott, ${ }^{49-51}$ a variation on the Selkov model of glycolysis, ${ }^{70}$ which has the form $f_{1}\left(u_{1}, u_{2}\right)=u_{1}-u_{1} u_{2}{ }^{2}+k_{1}(1$ $\left.-u_{1}\right), f_{2}\left(u_{1}, u_{2}\right)=u_{2}+u_{1} u_{2}{ }^{2}-\left(k_{1}+k_{2}\right) u_{2}$ where $k_{1}$ and $k_{2}$ are constants. Two-dimensional simulations reveal a variety of time-dependent and time-independent phenomena for various parameter values. The 'lamallae'-type patterns observed in ref. 46 and shown in Fig. 4 are found for some regions of the parameter space. The simulations also predicted the selfreplicating spots (see Plate 2) later found experimentally in ref. 46.

A four species presentation of the FIS reaction has been developed from an earlier model that considered ten species. ${ }^{71,72}$ Lee and Swinney ${ }^{73}$ have performed numerical simulations on the reduced model and compared results with experimental data. Numerical simulations show onedimensional phenomena that compare well with many of the experimental results concerned, including the lamallae and self-replication process. 

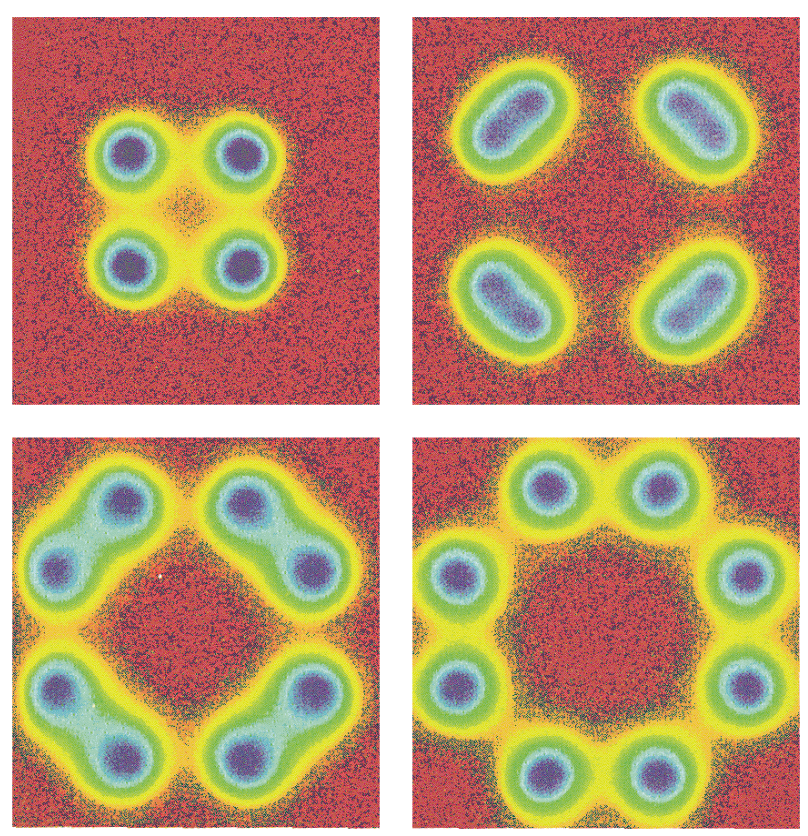

Plate 2 Time evolution of self-replicating spots generated by the numerical simulations in ref. 69. (Reprinted with kind permission from ref. 69.)

\section{Comparison of experimental results and theoretical predictions}

In Table 1 we present a comparison of Turing-type patterns observed in experiments with those observed in numerical simulations. The numerical simulations are not necessarily models of the specific reaction mentioned. This table is by no means exhaustive, but serves to provide a guide to some of the recent research in the area. (For example, it is known that many theoretical models can exhibit stripes, or spots or hexagonal structures. Here we list only a few examples.)
In the table we have used the following abbreviations: $\mathrm{PBC}=$ periodic boundary conditions; $0 \mathrm{FBC}=$ zero flux boundary conditions, IC $=$ initial conditions and $\mathrm{RIC}=$ random initial conditions

\section{Some open questions in chemical applications}

The theoretical study of spatial patterning in real chemical systems requires a highly reduced model of the full system. One example is the two-species model of the CIMA reaction developed by Lengyel and Epstein. While capturing basic elements of pattern formation, such models are unlikely to capture full features of the experimental system. Standard linear analysis of even a three-species RD model reveals a far greater array of patterning phenomena than the two-species model. ${ }^{76}$ Exploration of a more detailed RD model of the CIMA reaction may therefore reveal behaviour not previously reported in the reaction. Examples include the Turing-Hopf (or spatial-Hopf) bifurcation in which the resulting spatial patterns oscillate in both space and time, the interaction of Turing-Hopf and Turing-type patterns, and the interaction of two Turing-type patterns. The five-species model of the CIMA reaction may demonstrate phenomena of this nature and, subsequently, experimental study would be of great interest.

Some of the phenomena obtained experimentally have yet to be found in numerical simulations. An example is the black-eye patterns reported in the CIMA reaction. Although symmetry arguments have been used to describe these patterns theoretically, no numerical simulations have reported this feature as of date. Numerical simulations of these patterns would provide valuable information on the extent of occurrence.

The rhombic arrays have only been numerical simulated with other chemical kinetics, for example, Schnakenberg. ${ }^{2}$ Simulation of rhomboids with the Lengyel-Epstein model of the CIMA reaction may provide deeper understanding on how one can generate such patterns experimentally. It would also be of interest to examine whether such patterns can be formed 'naturally' rather than by imposing specific initial con-

Table 1 Comparison of experimental systems with computational models

\begin{tabular}{|c|c|c|}
\hline pattern & experimental systems & computational models \\
\hline hexagon $\left(H_{\pi}\right)$ & $\begin{array}{l}\text { CIMA reaction }{ }^{37,41} \\
\text { disc reactor } \\
\text { PA-MBO system } \\
\text { Petri dish }\end{array}$ & $\begin{array}{l}\text { Brandeisator model } \\
+\mathrm{PBC}+\mathrm{RIC}^{62}\end{array}$ \\
\hline $\begin{array}{l}\text { hexagon }\left(H_{0}\right) \\
\text { stripes }\end{array}$ & $\begin{array}{l}\text { PA-MBO system }{ }^{42} \\
\text { gel removed from the Petri dish } \\
\text { CIMA reaction }{ }^{37,41} \\
\text { disc reactor } \\
\text { PA-MBO system }{ }^{42} \\
\text { Petri dish }\end{array}$ & $\begin{array}{l}\text { Schnakenberg model } \\
+ \text { PBC + RIC } \\
\text { Brandeisator model } \\
+ \text { PBC + RIC }\end{array}$ \\
\hline rhombics & $\begin{array}{l}\text { CIMA reaction }{ }^{41} \\
\text { disc reactor } \\
\text { (1) + discontinuous change } \\
\text { of the control parameter } \\
\text { (2) + imposed rhombic pattern } \\
\text { as initial perturbation }\end{array}$ & $\begin{array}{l}\text { Schnakenberg model }{ }^{74} \\
\text { + PBC } \\
\text { + IC: vertical stripes with } \\
\text { oblique stripes added }\end{array}$ \\
\hline black-eyes & $\begin{array}{l}\text { CIMA reaction } \\
\text { disc reactor }\end{array}$ & none \\
\hline zigzags & $\begin{array}{l}\text { PA-MBO system } \\
\text { Petri dish }\end{array}$ & $\begin{array}{l}\text { Schnakenberg model }{ }^{74} \\
+ \text { PBC } \\
\text { + IC: vertical stripes with } \\
\text { oblique stripes added }\end{array}$ \\
\hline concentric ring & none & $\begin{array}{l}\text { Oregonator model }{ }^{75} \\
+0 \text { FBC } \\
+ \text { perturbation at centre }\end{array}$ \\
\hline mixed $H_{\pi}+$ stripe & $\begin{array}{l}\text { CIMA reaction }{ }^{37} \\
\text { disc reactor }\end{array}$ & $\begin{array}{l}\text { Schnakenberg model } \\
\text { transient pattern }\end{array}$ \\
\hline mixed $H_{0}+$ stripe & none & $\begin{array}{l}\text { Schnakenberg model } \\
\text { transient pattern }\end{array}$ \\
\hline
\end{tabular}


ditions. The demonstration of rhombic arrays with the Schnakenberg kinetics indicates that they may appear in a wide variety of chemical reactions that have the ability to develop Turing structures.

The role of boundary and initial conditions has received scant attention to date. Patterns can be highly influenced by the effect of different boundary conditions, especially when the length of the domain is of the order of a few wavelengths. Understanding how one can impose specific boundary conditions in experiments may provide greater understanding on the role of boundary conditions in determining pattern structure.

\section{Biological pattern formation}

Broadly speaking, there are two main models for spatial pattern formation in biology: (i) chemical pre-pattern models; and (ii) cell movement models. In (i), it is hypothesized that cells respond to a chemical pre-pattern and differentiate according to the concentration of chemical that they experience. The chemical pre-pattern may be set up by a simple source-sink mechanism, ${ }^{77}$ or by the more complicated RD mechanism. Although the pattern forming process in the former is simpler, the interpretation mechanism required by cells would be more complex than in the latter. ${ }^{78}$ In (ii), it is assumed that owing to mechanical forces and chemical cues, cells form a spatial pattern and cells in high density aggregate then differentiate. ${ }^{79-85}$

Both the above models, therefore, are based on fundamentally different biological assumptions. However, RD and mechanochemical models share many common features mathematically. Thus far, mechanochemical models have only been investigated in detail in one-dimension near primary bifurcation points and have been shown to exhibit patterns similar to those of the Turing models. Some limited two-dimensional analysis again reveals patterns similar to those observed in Turing models. More extensive investigation of the patterning properties of these models is required in order to determine if they can exhibit fundamentally different types of structures. Such predictions could then be used to differentiate experimentally between the two mechanisms.

Which mechanism operates is an area of huge controversy. In recent years certain candidates have been put forward as morphogens, most notably, retinoic acid, ${ }^{86}$ but its role in morphogenesis is still unclear. There are many examples where chemical patterns or cell aggregations are observed but it is still a question of great debate as to whether these are the cause of morphogenesis, or its effect. A detailed comparison and critical review of theoretical models for, and experimental results on, the skeletal pattern in the developing chick limb is given in ref. 87.

We concentrate, in this article, on Turing models for biological pattern formation. These models have been applied to diverse patterning phenomena too vast to be listed here, so we focus on only a few applications for which recent biological evidence suggests that the Turing model needs to be radically modified. One of the most widely studied applications has been to the patterning of body segments in the fruit fly Drosophila. ${ }^{5,88-93}$ Later numerical studies ${ }^{94}$ showed that the patterns produced by the model system were crucially sensitive to the geometry of the domain-slight perturbations in the shape of the domain resulted in a completely different pattern. This, of course, is a major drawback for the model application. More recently, detailed experimental work on Drosophila has shown that the pattern forming process is not, in fact, via reaction diffusion, but due to a cascade of gene switching, where certain gene proteins are expressed and, in turn, influence subsequent gene expression patterns. Therefore, although RD theory provides a very elegant mechanism for segmentation,

nature appears to have chosen a much less elegant way of doing it!

The formation of skeletal pattern in the limb has been the focus of a great deal of experimental and theoretical research. Recently, it has been shown that a number of Hox genes are switched on in a precise spatiotemporal manner in the developing chick limb. Although these are exciting advances, they still beg the question of how this patterning of activity is initiated. RD theory, as a model for the generation of such robust processes as digit formation, has been heavily criticised. For example, Bard and Lauder, ${ }^{95}$ showed that the qualitative form of the model solutions could be greatly influenced by minor perturbations in the system. In such an application, an essential requirement for a model is that it must be able to produce a limited number of patterns in a very robust way. In this respect, RD theory is too sophisticated because it exhibits a vast variety of patterns, many of which are never observed. Hence, one is forced to turn the question of pattern formation on its head and ask, how can one not generate so much pattern? We have addressed this issue by investigating the role of boundary conditions. The key point here is that certain types of boundary conditions preclude many patterns from forming while extending the domains of stability of the remaining patterns. This has been shown for an RD system in one dimension. ${ }^{96}$ Fig. 6 shows a comparison of the patterns formed under zero flux boundary conditions with conditions in which the boundary is a sink for one of the morphogens. The model now selects only patterns that are internal to the domain, and exhibits a patterning sequence that is consistent with that observed in the limb. The insight gained here then, is that the boundary plays an active role in the patterning mechanism, rather than simply being a passive impermeable membrane.

In 1990, Wolpert and Hornbruch ${ }^{97}$ performed an experiment in an attempt to prove that limb development in chick's could not possibly arise as a consequence of an RD or mechanochemical mechanism. They removed the posterior half of a host limb bud and replaced it by the anterior half of a donor limb bud so that the resultant double-anterior recombinant limb bud was the same size as a normal limb bud. This experiment was performed at a sufficiently early stage in development that no pattern was visible. The limbs developed two humeri instead of one. This contradicted both models, due to the fact that the model solutions are size-dependent, that is, if the domain size is unaltered, the patterns produced are unaltered.

In an attempt to rescue the theory, we assumed that the diffusion coefficient of the morphogens varied across the limb. The idea here was that, as diffusion and length scale are intimately linked, a varying diffusion coefficient essentially sets up an internal scaling, where the length scale in one part of the

(a)

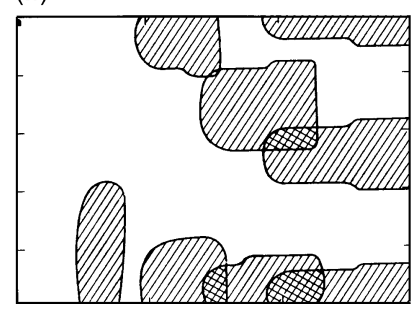

(b)

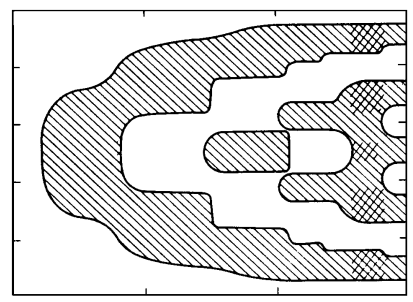

Fig. 6 Patterns produced by a reaction-diffusion system for different types of boundary conditions. In $(a)$, the boundary conditions are zero flux for each chemical and the model predicts patterns at the boundary. In $(b)$, one chemical is fixed to zero concentration at the boundary. In this case, the patterns form internal to the domain (see ref. 96 for details). (Reprinted with kind permission from ref. 96.) 
domain is so small that it cannot support Turing structures. Therefore, although combining two anterior halves results in a limb bud of normal size, it might actually consist of doubling the patterning sub-domain and hence in more complicated patterns. The prediction of the modelling was that there must be a variation of diffusion across the anterior-posterior axis of the chick limb. ${ }^{98,99}$ This actually turned out to be a 'postdiction' as experimental results show that gap junction permeability varies across the anterior-posterior axis of the chick limb. ${ }^{100,101}$

One of the most colourful applications of RD is in mammalian coat markings. ${ }^{102,103}$ In these applications, the qualitative features of the patterns do not change as the animal grows. Recently, Kondo and Asai ${ }^{104}$ considered the time evolution of skin patterns on the angelfish. On many species of this fish the juvenile exhibits vertical striped patterns. As it grows, the stripes move apart, and other stripes emerge between existing stripes to restore the original spatial wavelength of the patterning process. They showed that, at least on a one-dimensional domain, an RD model could exhibit patterns consistent with these observations. However, a numerical study of these equations on a two-dimensional domain reveals that these vertical stripes lose stability as the domain grows. ${ }^{105,106}$ Kondo and Asai, ${ }^{107}$ argue that there is an additional cue that selects vertical stripes rather than horizontal stripes or spots. However, the waters are muddied here by the observation that in most cases the adult does exhibit horizontal stripes or spots! This warrants more detailed investigation.

A crucial property of the patterns on the juvenile angelfish that was not addressed in ref. 104 and 105 is that the stripes that emerge between the existing stripes are thinner than the existing ones. This cannot be explained by a Turing mechanism. Recently, we have been considering a hybrid RD-cellmovement model wherein we assume that an RD mechanism sets up a pre-pattern in morphogen concentration, to which cells respond by moving chemotactically, that is, in response to chemical gradients (this is a well known phenomenon). Preliminary investigations on this model show that it can generate patterns with alternating thick and thin stripes. ${ }^{106}$

The final application we consider here also involves domain growth. It concerns the development of tooth primordia in the jaw of the alligator, Alligator mississippiensis. This is a complex process which has been the source of detailed experimental investigation, ${ }^{108-110}$ so there is ample experimental data on which to base a realistic mathematical model. As the jaw has left-right symmetry, one need consider only one side of the jaw. To a very good approximation, the jaw can be considered as one-dimensional. Teeth arise as the result of tooth primordia, which are clumps of cells in the jaw mesenchyme that mark where future teeth will form. The first seven tooth primordia form along the posterior-anterior axis in the sequence $7-3-6-2-5-1-4$. That is, the first primordium forms near the anterior end of the jaw, the second forms posterior to the first and the third forms posterior to the second. The fourth primordium, however, forms anterior to the first, and 5, 6 and 7 form in a posterior sequence. This complex sequence cannot be generated by the standard Turing model on a growing domain.

We have considered a modified Turing system which incorporates the biological observation that when a tooth primordium forms, it inhibits, for a certain length of time, neighbouring primordium formation. We consider a third chemical, a control chemical, which we assume is secreted by primordia and affects the morphogen kinetics. Numerical simulations show that this model exhibits spatiotemporal morphogen dynamics consistent with the pattern of primordia observed experimentally. ${ }^{111}$ To tie this in with the cell aggregations observed, we make the further hypothesis that cells respond to the morphogen pre-pattern by moving to areas of high morphogen concentration.

\section{Some open questions in biological applications}

Most theoretical work on RD theory has focused on solving the system on a fixed one-dimensional domain with zero-flux boundary conditions and constant parameters. This is the easy case but only scratches the surface of the study of these systems. In a biological context, parameters themselves will vary over space and time, boundary conditions will be more complicated than zero flux and two crucial differences to the case of the chemical systems discussed above are that the domains do not have simple geometry and that they grow. Although RD theory has been around as a model for morphogenesis for over 40 years, there are precious few cases in which the model has been shown to exhibit patterns consistent with those observed in nature for the case of realistic geometry and domain growth in two and three dimensions. This is remarkable when one considers that the first necessary step for any model is that it must be shown to exhibit the phenomena it purports to model. Of course, part of the problem has been the lack of computer power necessary to carry out a thorough numerical investigation of the model.

It has been shown recently that the form of the nonlinearity in the chemical kinetics determines whether the model exhibits spots or stripes. ${ }^{112-114}$ Other key questions to address are the effects of different types of non-linear kinetics, boundary conditions, domain growth, and domain geometry, on the selection and stabilization of patterns.

On the biological side, the crucial question still concerns the unequivocal identification of morphogens. We still seem a long way from that goal. It is also necessary for the theory, where possible, to make experimentally testable predictions.

\section{Conclusion}

The recent discovery of spatial pattern formation in chemistry, some 40 years after Turing's prediction, has revitalised the field and led to a great deal of interest in the properties of Turing models. Biology is still lagging behind, which is hardly surprising as it is on yet another level of complexity. However, the application of Turing models to biological pattern formation, and the interaction between model predictions and experimental observations, have led to model modifications that, in turn, suggest new insights to the underlying biological mechanisms at work. With the enormous advances in biotechnology and computer power, the field seems poised on a threshold of new discoveries.

K.J.P. acknowledges receipt of an Engineering and Physical Sciences Research Committee (EPSRC) research studentship. We thank an anonymous referee for a number of very helpful comments including the analogy on page 1 . We also thank Len Fisher for helpful suggestions.

\section{References}

1 A. M. Turing, Philos. Trans. R. Soc. London, Sect. B, 1952, 327, 37.

2 J. Schnakenberg, J. Theor. Biol., 1979, 81, 389.

3 P. Fife, Mathematical Aspects of Reacting and Diffusing Systems, Lecture Notes in Biomathematics, Springer-Verlag, Berlin, Heidelberg, New York, 1979, vol. 28.

4 J. D. Murray, Mathematical Biology, Springer-Verlag, Berlin, Heidelberg, New York, 1993.

5 H. Meinhardt, Models of Biological Pattern Formation, Academic Press, London, 1982.

6 L. Edelstein-Keshet, Mathematical Models in Biology, Random House, New York, 1988.

7 N. F. Britton, Reaction-Diffusion Equations and Their Applications to Biology, Academic Press, London, 1986.

8 L. A. Segel, Modeling Dynamic Phenomena in Molecular and Cell Biology, Cambridge University Press, 1989.

9 L. A. Segel and J. L. Jackson, J. Theor. Biol., 1972, 37, 545.

10 Y. I. Balkarei, A. V. Grigor'Yants, Y. A. Rzhanov and M. I. Elinson, Opt. Commun., 1988, 66, 16. 
11 Self-Organization, Auto-Waves and Structures Far From Equilibrium, ed. V. I. Krinski, Springer, Berlin, 1984.

12 D. Walgraef and N. M. Ghoniem, Phys. Rev. B, 1990, 13, 8867.

13 D. B. White, J. Fluid Mech., 1988, 191, 247.

14 T. Nozakura and S. Ikeuchi, Astrophys. J., 1984, 279, 40

15 M. Maruyama, Am. Sci., 1963, 51, 164

16 B. P. Belousov, in Oscillations and Travelling Waves in Chemical Systems, ed. R. J. Field and M. Burger, Wiley, New York, 1985, p. 605.

17 Oscillations and Travelling Waves in Chemical Systems, ed. R. J. Field and M. Burger, Wiley, New York, 1985.

18 B. R. Johnson and S. K. Scott, Chem. Soc. Rev., 1996, 265.

19 R. J. Field, E. Körös and R. M. Noyes, J. Am. Chem. Soc., 1972, 94, 8649.

20 R. J. Field and R. M. Noyes, J. Chem. Phys., 1974, 60, 1877.

21 J. J. Tyson, The Belousov-Zhabotinskii Reaction, Lecture Notes in Biomathematics, Springer-Verlag, Berlin, Heidelberg, New York, 1976, vol. 10

22 J. J. Tyson, in Oscillations and Travelling Waves in Chemical Systems, ed. R. J. Field and M. Burger, Wiley, New York, 1985, p. 92 .

23 S. K. Scott, Chemical Chaos, Oxford University Press, 1991.

24 A. N. Zaikin and A. M. Zhabotinskii, Nature (London), 1970, 225, 535 .

25 A. T. Winfree, Science, 1972, 175, 634.

26 A. T. Winfree, Science, 1973, 181, 937.

27 A. T. Winfree, SIAM-AMS Proc., 1974, 8, 13.

28 A. T. Winfree, Theor. Chem., 1978, 4, 1.

29 S. C. Müller, T. Plesser and B. Hess, Science, 1985, 230, 661.

30 B. J. Welsh, J. Gomatam and A. E. Burgess, Nature (London), 1983, 304, 611.

31 A. B. Rovinskii, J. Chem. Phys., 1987, 91, 4606

32 G. Nicolis and I. Progogine, Self-Organization in Nonequilibrium Systems, Wiley, New York, 1977.

33 J. Pearson and W. Horsthemcke, J. Chem. Phys., 1989, 90, 1588.

34 V. Castets, E. Dulos, J. Boissonade and P. De Kepper, Phys. Rev. Lett., 1990, 64, 2953.

35 P. De Kepper, V. Castets, E. Dulos and J. Boissonade. Physica D, 1991, 49, 161.

36 P. De Kepper, I. R. Epstein, K. Kustin and M. Orbán, J. Phys. Chem., 1982, 86, 170.

37 Q. Ouyang and H. L. Swinney, Nature (London), 1991, 352, 610.

38 Q. Ouyang and H. L. Swinney, Chaos, 1991, 1, 411.

39 D. Walgraef, G. Dewel and P. Borckmans, Adv. Chem. Phys., 1982, 49, 311.

40 Q. Ouyang, Z. Noszticius and H. L. Swinney, J. Phys. Chem., $1992,96,6773$

41 G. H. Gunaratne, Q. Ouyang and H. L. Swinney, Phys. Rev. E, 1994, 50, 2802.

42 M. Watzl and A. F. Münster, Chem. Phys. Lett., 1995, 242, 273.

43 M. Burger and R. J. Field, Nature (London), 1984, 307, 720.

44 P. Resch, R. J. Field and F. W. Schneider, J. Phys. Chem., 1989, 93, 2783.

45 Y. X. Zhang and R. J. Field, J. Phys. Chem., 1991, 95, 723.

46 K. Lee, W. D. McCormick, Q. Ouyang and H. L. Swinney, Science, 1993, 261, 192

47 K. Lee, W. D. McCormick, J. E. Pearson and H. L. Swinney, Nature (London), 1994.

48 W. N. Reynolds, J. E. Pearson and S. Ponce-Dawson, Phys. Rev. Lett., 1994, 72, 2797.

49 P. Gray and S. K. Scott, Chem. Eng. Sci., 1983, 38, 29.

50 P. Gray and S. K. Scott, Chem. Eng. Sci., 1984, 39, 1087.

51 P. Gray and S. K. Scott, J. Phys. Chem., 1985, 89, 22.

52 D. Haim, G. Li, Q. Ouyang, W. D. McCormick, H. L. Swinney, A. Hagberg and E. Meron, Phys. Rev. Lett., 1996, 77, 190.

53 I. Lengyel and I. R. Epstein, Proc. Natl. Acad. Sci. USA, 1992, 89, 3977.

54 I. R. Epstein and I. Lengyel, Physica D, 1995, 84, 1.

55 A. Gierer and H. Meinhardt, Kybernetik, 1972, 12, 30

56 D. Thomas, in Analysis and Control of Immobilized Enzyme Systems, ed. D. Thomas and J.-P. Kernevez, Springer, Berlin, Heidelberg, New York, 1975, p. 115.

57 V. Dufiet and J. Boissonade, Physica A, 1992, 188, 158.

58 I. Lengyel, G. Rabai and I. R. Epstein, J. Am. Chem. Soc., 1990, 112, 4606.

59 I. Lengyel, G. Rabai and I. R. Epstein, J. Am. Chem. Soc., 1990, 112, 9104

60 I. Lengyel and I. R. Epstein, Science, 1991, 251, 650.

61 I. Lengyel and I. R. Epstein, Acc. Chem. Res., 1993, 26, 235.

62 O. Jensen, E. Mesekilded, P. Borckmans and G. Dewel, Phys. Scr., 1996, 53, 243.
63 K. Lee, W. D. McCormick, H. L. Swinney and Z. J. Noszticius, J. Chem. Phys., 1992, 95, 4048.

64 K. Agladze, E. Dulos and P. De Kepper, J. Phys. Chem., 1992 96, 2400 .

65 Z. Noszticius, Q. Ouyang, W. D. McCormick and H. L. Swinney, J. Phys. Chem., 1992, 96, 6302.

66 J. J. Perraud, A. De Wit, E. Dulos, P. De Kepper, G. Dewel and P. Borckmans, Phys. Rev. Lett., 1993, 71, 1272.

67 R. Kapral, Physica D, 1995, 86, 149.

68 Q. Ouyang, R. Li, G. Li and H. L. Swinney, J. Chem. Phys., 1995, 102, 2551.

69 J. E. Pearson, Science, 1993, 261, 189.

70 E. E. Selkov, Eur. J. Biochem., 1968, 4, 79

71 V. Gaspar and K. Showalter, J. Am. Chem. Soc., 1987, 109, 4876.

72 V. Gaspar and K. Showalter, J. Phys. Chem., 1990, 94, 4973.

73 K. Lee and H. L. Swinney, Phys. Rev. E, 1995, 51, 1899.

74 V. Dufiet and J. Boissonade, J. Chem. Phys., 1992, 96, 664

75 J. Guslander and R. J. Field, J. Phys. Chem., 1992, 96, 10575.

76 H. G. Othmer and L. E. Scriven, Ind. Eng. Chem. Fundam., 1969, 8, 302.

77 L. Wolpert, J. Theor. Biol., 1969, 25, 1.

78 B. N. Nagorcka, J. Theor. Biol., 1989, 137, 127.

79 G. F. Oster, J. D. Murray and A. K. Harris, J. Embryol. Exp. Morphol., 1983, 78, 83.

80 J. D. Murray and G. F. Oster, IMA J. Math. Appl. Med. Biol., 1984, 1, 51.

81 J. D. Murray and G. F. Oster, J. Math. Biol., 1984, 19, 265.

82 G. F. Oster, J. D. Murray and P. K. Maini, J. Embryol. Exp. Morphol., 1985, 89, 93.

83 P. K. Maini, M. R. Myerscough, J. D. Murray, K. H. Winters, Bull. Math. Biol., 1991, 53, 701.

84 J. D. Murray, D. C. Deeming and M. W. J. Ferguson, Proc. R. Soc. London, Ser. B, 1990, 239, 1990.

85 J. D. Murray and M. R. Myerscough, J. Theor. Biol., 1991, 149, 339.

86 C. Thaller and G. Eichele, Nature (London), 1987, 327, 625.

87 P. K. Maini and M. Solursh, Int. Rev. Cytol., 1991, 129, 91.

88 S. A. Kauffman, R. Shymko and K. Trabert, Science, 1978, 199 259.

89 S. A. Kauffman, Philos. Trans. R. Soc. London B, 1981, 295, 567.

90 T. C. Lacalli, D. A. Wilkinson and L. G. Harrison, Development, 1988, 103, 105.

91 T. C. Lacalli, J. Theor. Biol., 1990, 144, 171

92 H. Meinhardt, J. Cell Sci., 1977, 23, 117.

93 H. Meinhardt, J. Cell Sci. Suppl., 1986, 4, 357.

94 B. Bunow, J.-P. Kernevez, G. Joly and D. Thomas, J. Theor. Biol., 1980, 84, 629

95 J. Bard and I. Lauder, J. Theor. Biol., 1974, 45, 501.

96 R. Dillon, P. K. Maini and H. G. Othmer, J. Math. Biol., 1994 32, 345.

97 L. Wolpert and A. Hornbruch, Development, 1990, 109, 961.

98 P. K. Maini, D. L. Benson and J. A. Sherratt, IMA J. Math Appl. Med. Biol., 1992, 9, 197.

99 P. K. Maini, J. Biol. Syst., 1995, 3, 987.

100 F. Brümmer, G. Zempel, P. Buhle, J.-C. Stein and D. F. Hulser, Exp. Cell. Res., 1991, 196, 158

101 C. N. D. Coelho and R. A. Kosher, Dev. Biol., 1991, 148, 529.

102 J. D. Murray, J. Theor. Biol., 1981, 88, 161.

103 J. D. Murray, Sci. Am., 1988, 258, 80.

104 S. Kondo and R. Asai, Nature (London), 1995, 376, 765.

105 T. Höfer and P. K. Maini, Nature (London), 1996, 380, 678.

106 K. Painter, P. K. Maini and H. G. Othmer, in preparation.

107 S. Kondo and R. Asai, Nature (London), 1996, 380, 678.

108 B. Westergaard and M. W. J. Ferguson, J. Zool. London, 1986, 210, 575.

109 B. Westergaard and M. W. J. Ferguson, J. Zool. London, 1987, 212, 191.

110 B. Westergaard and M. W. J. Ferguson, Am. J. Anatomy, 1990, 187, 393.

111 P. M. Kulesa, G. C. Cruywagen, S. R. Lubkin, P. K. Maini, J. Sneyd, M. W. J. Ferguson and J. D. Murray, J. Theor. Biol., 1996, 180, 287.

112 B. Ermentrout, Proc. R. Soc. London, Ser. A, 1991, 434, 413

113 B. N. Nagorcka and J. R. Mooney, IMA J. Math. Appl. Med. Biol., 1992, 9, 249.

114 M. J. Lyons and L. G. Harrison, Dev. Dyn., 1992, 195, 201.

Paper 7/02602A; Received 16th April, 1997 